\title{
BAIRE SPACES, $k$-SPACES, AND SOME PROPERLY HEREDITARY PROPERTIES
}

\author{
ADNAN AL-BSOUL
}

Received 11 October 2004 and in revised form 8 September 2005

A topological property is properly hereditary property if whenever every proper subspace has the property, the whole space has the property. In this note, we will study some topological properties that are preserved by proper subspaces; in fact, we will study the following topological properties: Baire spaces, second category, sequentially compact, hemicompact, $\delta$-normal, and spaces having dispersion points. Also, we will solve some open problems raised by Al-Bsoul (2003) and Arenas (1996) and conclude this note by some open problems.

\section{Introduction}

In 1996, Professor M. L. Puertas suggested the following question which was studied by Arenas in his paper [2]:

(*) if every proper subspace has the property, the whole space has the property.

Any topological property satisfying the property $(*)$ is called properly hereditary property. Moreover, if every proper closed (resp., open, $F_{\sigma}, G_{\delta}$, etc.) subspace has the property, then the whole space has the property, and we call such a property properly closed (resp., open, $F_{\sigma}, G_{\delta}$, etc.) hereditary.

In [2], Arenas studied some topological properties having the property $(*)$. In fact, Arenas [2] proved that the topological properties $T_{i}, i=0,1,2,3$, are properly hereditary properties. He also studied countability axioms and metrizability. Nevertheless, for Arenas, the property $\left(^{*}\right)$ makes sense only for the topological properties that are hereditary to all subsets. On the other hand, as we saw in [1], there are some topological properties which are properly hereditary properties but are not hereditary properties, for instance, paracompactness. Thus, we may ask the converse.

Question 1.1. Is there a topological property $P$ which is hereditary but is not properly hereditary?

It is obvious to see that if a topological property is properly hereditary, it is not necessary that it is properly open (closed) hereditary. So, it is natural to redefine the following (compare with the definition in [2]). 
Definition 1.2. A topological property is called strong (resp., closed, open, $F_{\sigma}, G_{\delta}$, etc.) hereditary property provided that it is (resp., closed, open, $F_{\sigma}, G_{\delta}$, etc.) hereditary property and properly (resp., closed, open, $F_{\sigma}, G_{\delta}$, etc.) hereditary property.

Hence, $T_{i}$ is a strong hereditary property for $i=0,1 / 4,1 / 2,1,1(1 / 3), 2,2(1 / 2), 3$, but $T_{1}, T_{1(1 / 3)}, T_{2}, T_{2(1 / 2)}, T_{3}$ are neither strong open hereditary properties nor strong closed hereditary properties.

In [1], Al-Bsoul studied some topological properties as some nonfamiliar separation axioms beside some strong separation axioms, and also some covering properties.

Reading [1, 2], the reader may conclude that all classical topological properties are properly hereditary properties. This is not the case; however, in this note we are going to give two topological properties which are not properly (resp., closed, open) hereditary properties. Moreover, we will positively solve two open problems raised in $[1,2]$. Also, we will prove that some topological properties, such as sequential compactness, hemicompactness, and $\delta$-normality, are properly hereditary properties.

\section{Baire spaces and $k$-spaces}

In this section we will positively solve the following open problem raised in [1].

Question 2.1 [1]. Are Baire spaces and second category properly hereditary properties?

Theorem 2.2. If every proper subspace of $X$ is a Baire subspace, then $X$ is a Baire space.

Proof. Let $\left\{G_{n}: n \in \mathbb{N}\right\}$ be a countable family of open dense subsets of $X$. Pick $x_{0} \in$ $X \backslash\left(\bigcap_{n \in \mathbb{N}} G_{n}\right)$. If such $x_{0}$ does not exist, then $\bigcap_{n \in \mathbb{N}} G_{n}=X$, and the proof is finished.

Let $Y=X \backslash\left\{x_{0}\right\}$, then $G_{n} \backslash\left\{x_{0}\right\} \neq \Phi$ for all $n \in \mathbb{N}$. If ${\overline{G_{i} \backslash\left\{x_{0}\right\}}}^{Y} \neq Y$ for some $i \in \mathbb{N}$, then there exist $t \in Y \backslash\left({\overline{G_{i} \backslash\left\{x_{0}\right\}}}^{Y}\right)$ and an open set $V$ in $Y$ such that $t \in V \subseteq Y \backslash\left(\overline{G_{i} \backslash\left\{x_{0}\right\}}{ }^{Y}\right)$. So, there exists an open set $U$ in $X$ such that $V=U \cap Y$. Now, $U \cap G_{i} \neq \Phi$ and $U=V \cup\left\{x_{0}\right\}$, so $V \cap G_{i}=\Phi$, and hence $U \cap G_{i}=\left\{x_{0}\right\}$, which is a contradiction. Thus $G_{n} \backslash\left\{x_{0}\right\}$ is an open dense set in $Y$ for all $n \in \mathbb{N}$. Therefore, $\left(\bigcap_{n \in \mathbb{N}} G_{n}\right)$ is dense in $X$.

Corollary 2.3. Let $X$ be a $T_{1}$-space. If every proper open subspace of $X$ is a Baire subspace, then $X$ is a Baire space.

\section{Corollary 2.4. Second category is properly hereditary property.}

Proof. Let $X$ be a space such that every proper subspace of $X$ is a second category, and let $\left\{G_{n}: n \in \mathbb{N}\right\}$ be a countable family of open dense subsets of $X$. Suppose that $\bigcap_{n \in \mathbb{N}} G_{n}=$ $\Phi$. Pick $x_{0} \in X$, and let $Y=X \backslash\left\{x_{0}\right\}$, then $G_{n} \backslash\left\{x_{0}\right\}$ is an open dense subset of $Y$ for all $n \in \mathbb{N}$. Therefore, $\bigcap_{n \in \mathbb{N}} G_{n} \neq \Phi$.

Also, we will solve the following open problem for $k$-spaces positively.

Question 2.5 [2]. Is being a $k$-space a properly (closed) hereditary property?

Recall that a Hausdorff space $X$ is called a $k$-space if and only if the following condition holds:

$A \subseteq X$ is open if $A \cap K$ is open in $K$ for each compact set $K$ in $X$. 
In [2, Example 2.2], we see that every proper closed subspace of $X$ is a $k$-subspace, but $X$ itself is not a $k$-space. Also, [1, Example 1.1], shows that every proper open subspace of $X$ is a $k$-subspace, but $X$ itself is not a $k$-space. But we have the following theorem.

Theorem 2.6. If every proper subspace of $X$ is a $k$-subspace, then $X$ is a $k$-space.

Proof. Let $U$ be an open subset in $X$ and let $K$ be any compact subset in $X$. If $U \subseteq K$ or $K \subseteq U$, we are done. Assume that $U \backslash K \neq \Phi$ and $K \backslash U \neq \Phi$. Fix a point $x_{0} \in U \backslash K$, then $U \cap K=\left(U \backslash\left\{x_{0}\right\}\right) \cap K$ is open in $K$.

Conversely, Assume that $U \cap K$ is open in $K$ for each compact set $K$ in $X$. If $U=X$ or $U=\Phi$, we are done. Assume that $U$ is a nonempty proper subset of $X$, so choose $y_{0} \in X \backslash U$, and let $Y=X \backslash\left\{y_{0}\right\}$, so $U \cap K_{Y}$ is open in $K_{Y}$ for each compact set $K_{Y}$ in $Y$, so $U$ is open in $Y$ and hence in $X$.

Corollary 2.7. For Hausdorff spaces, if every proper closed (resp., open) subspace of $X$ is a $k$-subspace, then $X$ is a $k$-space.

\section{More topological properties satisfying $(*)$}

Several topological properties will be studied in this section. Let us start with some types of compactness.

THeOREM 3.1. $\sigma$-compactness is a properly hereditary property.

Theorem 3.2. Sequential compactness is a properly hereditary property.

Proof. Let $\left\{x_{n}\right\}$ be a sequence in $X$. If $\left|\left\{x_{n}: n \in \mathbb{N}\right\}\right|<\mathfrak{N}_{0}$, then it has a constant subsequence. If the value $x_{n_{0}}$ is repeated infinitely many times, that is, the set $\left\{n: x_{n}=x_{n_{0}}\right\}$ is infinite, then this sequence has a constant subsequence. If $x_{1}$ is repeated only finitely many times, assume that $x_{n} \neq x_{1}$ for all $n>n_{0}$, hence the subsequence $x_{n_{j}}=x_{n_{0}+j}$ has a convergent subsequence in $X \backslash\left\{x_{1}\right\}$ and hence in $X$.

Since the following topological property (hemicompact or denumerable at infinity) is not familiar, we give its definition.

Definition 3.3. A Hausdorff space $X$ is said to be hemicompact or denumerable at infinity if and only if there is a sequence $K_{1}, K_{2}, \ldots$ of compact subsets of $X$ such that if $K$ is any compact subset of $X$, then $K \subseteq K_{n}$ for some $n$.

TheоRем 3.4. If every proper subspace of $X$ is a hemicompact subspace, then $X$ is a hemicompact space.

Proof. Let $x \neq y$ in $X$, so there exists an open set $V$ in $X$ such that $x \notin \bar{V}$. Since $Y=X \backslash V$ and $\bar{V}$ are hemicompact, there exist compact subsets $C_{1}, C_{2}, \ldots$ of $Y$, such that for every compact subset $K_{Y}$ of $Y$ there exists $C_{n_{0}}$ such that $K_{Y} \subseteq C_{n_{0}}$, and there exist compact subsets $D_{1}, D_{2}, \ldots$ of $\bar{V}$, such that for every compact subset $K_{\bar{V}}$ of $\bar{V}$ there exists $D_{m_{0}}$ such that $K_{\bar{V}} \subseteq D_{m_{0}}$. Let $W=\{0\} \cup \mathbb{N}$, and define a sequence of compact subsets $K_{m, n}$ of $X$ as follows: $K_{m, n}=C_{m} \cup D_{n}, K_{m, 0}=C_{m}$, and $K_{0, n}=D_{n}$ for all $(m, n) \in W \times W \backslash\{(0,0)\}$.

Now, if $K$ is any compact subset of $X$, then $K \cap Y$ is compact in $Y$ and $K \cap \bar{V}$ is compact in $\bar{V}$. Thus, there exist $C_{m_{0}}$ and $D_{n_{0}}$ such that $K \cap Y \subset C_{m_{0}}$ and $K \cap \bar{V} \subset D_{n_{0}}$, hence $K=(K \cap Y) \cup(K \cap \bar{V}) \subset C_{m_{0}} \cup D_{n_{0}}=K_{m_{0}, n_{0}}$. 
It is easy to construct examples to show that hemicompactness is neither a properly open hereditary property nor a properly closed hereditary property. Recall that a space $X$ is said to be $\delta$-normal if whenever $A$ and $B$ are disjoint closed subsets of $X$, there exist two disjoint $G_{\delta}$-sets $H$ and $K$ such that $A \subseteq H$ and $B \subseteq K$.

Example 1.1 in [1], shows that if every closed proper subspace of $X$ is a $\delta$-normal $T_{1}$ subspace, then $X$ need not be a $\delta$-normal space. Moreover, [2, Example 2.2] shows that if every open proper subspace of $X$ is a $\delta$-normal $T_{1}$-subspace, then $X$ need not be a $\delta$-normal space. Fortunately, we have the following result.

Theorem 3.5. If every proper subspace of $X$ is a $\delta$-normal $T_{1}$-subspace, then $X$ is a $\delta$-normal space.

Proof. Let $A$ and $B$ be two nonempty disjoint closed subsets of $X$. The case $X=A \cup B$ is evident. Let $y_{0} \in X \backslash(A \cup B)$, and let $Y=X \backslash\left\{y_{0}\right\}$, so there exist two disjoint $G_{\delta}$-sets $H_{0}, K_{0}$ in $Y$ such that $A \subseteq H_{0}$ and $B \subseteq K_{0}$, thus $H_{0}=\bigcap_{i=1}^{\infty} H_{i}$ and $K_{0}=\bigcap_{i=1}^{\infty} K_{i}$, where $H_{i}$ and $K_{i}$ are open in $Y$ for all $i \in \mathbb{N}$. Hence, there exist open sets $U_{i}$ and $V_{i}$ in $X$, such that $H_{i}=U_{i} \cap Y$ and $K_{i}=V_{i} \cap Y$ for all $i \in \mathbb{N}$. Thus, $U=\bigcap_{i=1}^{\infty} U_{i}$ and $V=\bigcap_{i=1}^{\infty} V_{i}$ are $G_{\delta^{-}}$ sets. If $U \cap V=\Phi$, the proof is completed, otherwise, $U \cap V=\left\{y_{0}\right\}$. Take $z_{0} \in X \backslash(A \cup$ $B \cup\left\{y_{0}\right\}$ ), and let $Z=X \backslash\left\{z_{0}\right\}$, so there exist two disjoint $G_{\delta}$-sets $H^{0}, K^{0}$ in $Z$ such that $A \subseteq H^{0}$ and $B \subseteq K^{0}$, thus $H^{0}=\bigcap_{i=1}^{\infty} H^{i}$ and $K^{0}=\bigcap_{i=1}^{\infty} K^{i}$, where $H^{i}$ and $K^{i}$ are open in $Z$ for all $i \in \mathbb{N}$. Hence, there exist open sets $U^{i}$ and $V^{i}$ in $X$, such that $H^{i}=U^{i} \cap Z$ and $K^{i}=V^{i} \cap Z$ for all $i \in \mathbb{N}$. Let $L_{i}=U_{i} \cap U^{i}$ and $M_{i}=V_{i} \cap V^{i}$, and define the required $G_{\delta}$-sets $L=\bigcap_{i=1}^{\infty} L_{i}$ and $M=\bigcap_{i=1}^{\infty} M_{i}$.

It is easy to prove the following.

Theorem 3.6. Pseudocompactness is a properly hereditary property.

Recall that a space $X$ is extremely disconnected if and only if the closure of every open set is open. This topological property is not a properly (resp., closed, open) hereditary property, as we will see in the next example.

Example 3.7. Let $X=\{u, x, y\}$ and let $\tau=\{\{u\},\{y\},\{u, y\}, \Phi, X\}$, hence every proper subspace of $X$ is an extremely disconnected subspace, but $X$ itself is not.

Theorem 3.8. The topological property "extremely disconnected" is a properly hereditary property in the class of $T_{1}$-spaces.

Remark 3.9. If every proper open subspace of $X$ is an extremely disconnected $T_{1}$ subspace, then $X$ is not necessarily an extremely disconnected space.

\section{Open problems}

In this section, we will study a special kind of topological properties, namely, having a dispersion point. For this, let us state its definition. Recall that a connected space $X$ is said to have a dispersion point $p$ if and only if $X \backslash\{p\}$ is totally disconnected. Thus, no space $X$ of cardinality greater than 2 has the property that every subspace of $X$ has a dispersion point because, if $x, y \in X \backslash\{p\}$, where $p$ is the dispersion point of $X$, then the subspace $\{x, y\}$ has the discrete topology, and hence has no dispersion point. Thus, it is natural to ask the following question. 
Question 4.1. Is there a connected space for which every proper open (closed) subspace of $X$ has a dispersion point?

Indeed, take [2, Example 2.2] under consideration for the case of proper open subspaces, and take [1, Example 1.1] for the case of proper closed subspaces. Thus, one may ask the following.

Question 4.2. Is the property of having a dispersion point a properly open (closed) hereditary property?

The next example is a modification of [1, Example 1.1], which shows that the answer of Question 4.2 is negative in general.

Example 4.3. Let $X=\mathbb{Z} \cup\{\pi, \sqrt{2}\}$, and topologize $X$ as

$$
\tau=\{A \cup\{\pi\}: A \subseteq \mathbb{Z}\} \cup\{X, \Phi\} .
$$

So, $\pi$ is a dispersion point of every nonempty proper open subspace of $X$, and $\sqrt{2}$ is a dispersion point of every nonempty proper closed subspace of $X$. But $X$ itself has no dispersion points.

Note that the space in Example 4.3 is not a $T_{1}$-space, but it is easy to see that there is no $T_{1}$-space for which every proper closed (open) subspace has a dispersion point. A topological space $X$ is called $T_{1 / 2}$ if and only if every singleton is open or closed. Also, a topological space $X$ is called $T_{1 / 4}$ if and only if for every finite subset $F$ of $X$ and for every $y \in X \backslash F$, there exists a set $A$ containing $F$ and not containing $y$ which is either open or closed in $X$. Hence we can restate the previous questions as follows.

Question 4.4. Is there a $T_{1 / 4}\left(T_{1 / 2}\right)$-space for which every proper open (closed) subspace of $X$ has a dispersion point? If so, does $X$ have a dispersion point?

Questions 1.1 and 4.4 are still open.

For the following two questions, the reader may consult [3].

Question 4.5. Is maximal compactness (minimal Hausdorffness, realcompactness, absolutely countably compactness) a properly (resp., closed, open, $F_{\sigma}$, $G_{\delta}$, etc.) hereditary property?

Question 4.6. Is $H$-closedness a properly (resp., closed, open, $F_{\sigma}$, $G_{\delta}$, etc.) hereditary property?

\section{References}

[1] A. Al-Bsoul, Some separation axioms and covering properties preserved by proper subspaces, Questions Answers Gen. Topology 21 (2003), no. 2, 171-175.

[2] F. G. Arenas, Topological properties preserved by proper subspaces, Questions Answers Gen. Topology 14 (1996), no. 1, 53-57.

[3] S. Willard, General Topology, Addison-Wesley, Massachusetts, 1970.

Adnan Al-Bsoul: Department of Mathematics and Physics, Faculty of Arts and Science, Qatar University, P.O. Box 2713, Doha, Qatar

E-mail addresses: albsoulaaa@hotmail.com; albsoulaaa@qu.edu.qa 


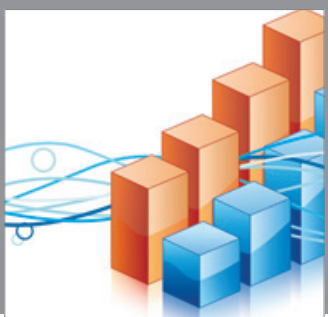

Advances in

Operations Research

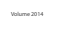

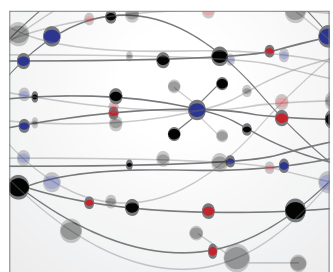

\section{The Scientific} World Journal
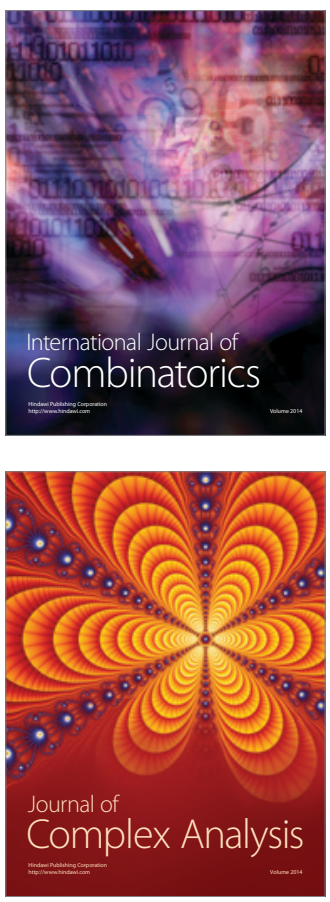

International Journal of

Mathematics and

Mathematical

Sciences
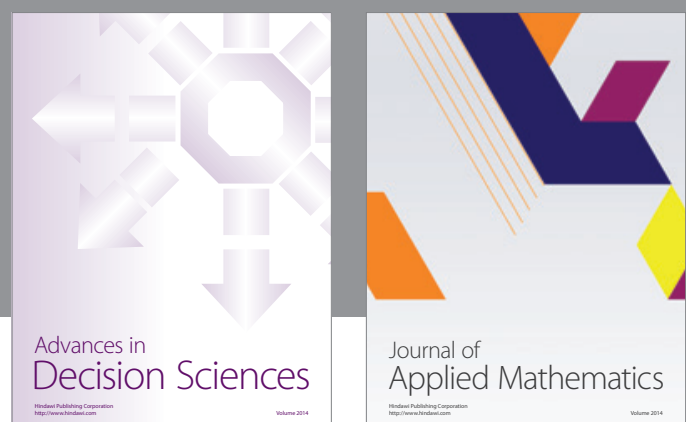

Journal of

Applied Mathematics
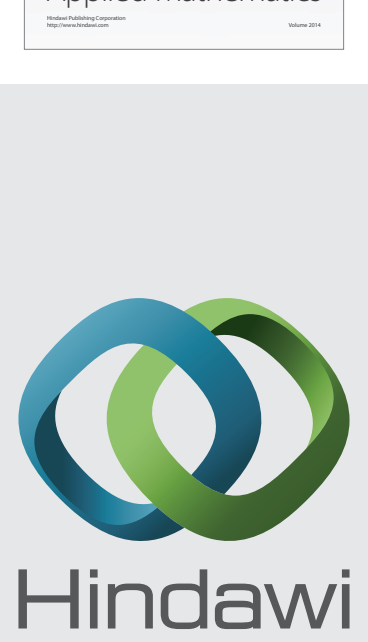

Submit your manuscripts at http://www.hindawi.com
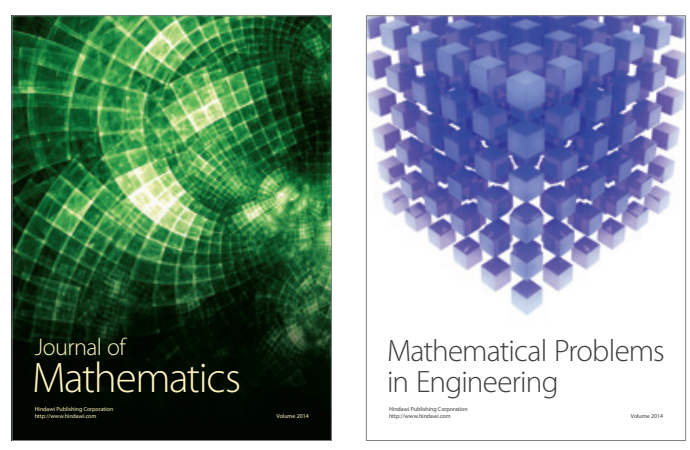

Mathematical Problems in Engineering
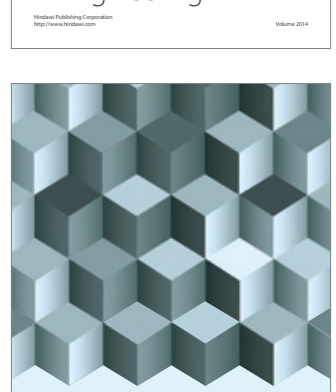

Journal of

Function Spaces
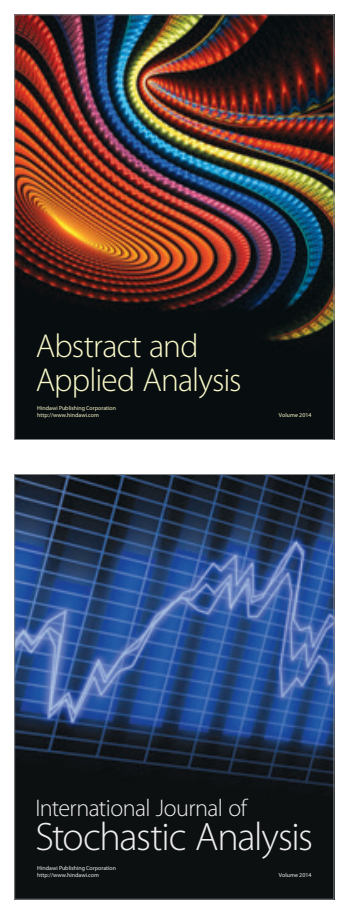

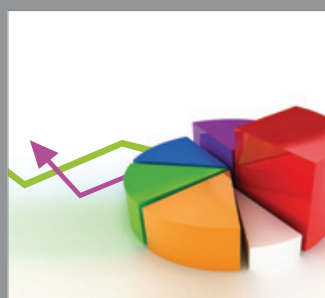

ournal of

Probability and Statistics

Promensencen
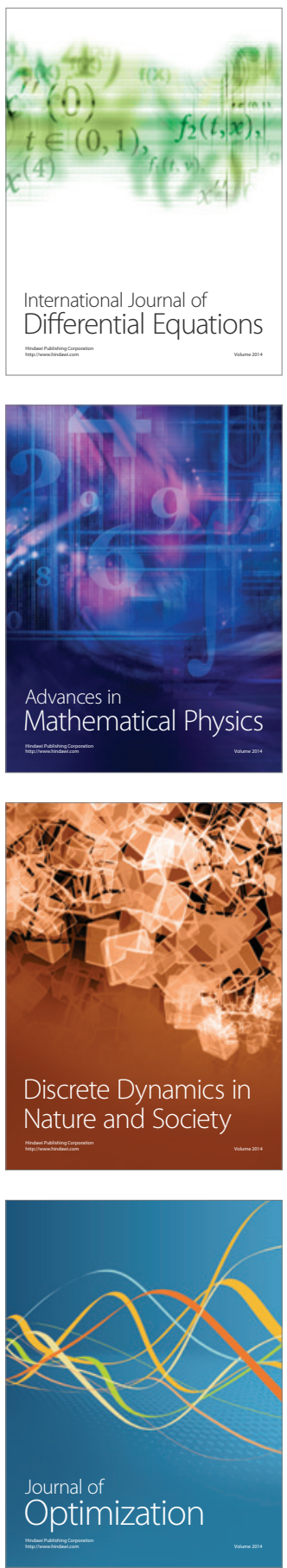